\title{
Triptolide mediates Wnt/ $\beta$-catenin signalling pathway to reduce cerebral ischemia-reperfusion injury in rats
}

\author{
Wenyong Pan $^{1}$, Zhiming $\mathrm{Xu}^{2}$ \\ ${ }^{1}$ Department of Neurosurgery, Qingdao Chengyang People's Hospital, Qingdao, China, ${ }^{2}$ Department of Neurosurgery, \\ Qingdao Municipal Hospital, Qingdao, China
}

\begin{abstract}
Introduction: Triptolide, extracted from Chinese medicinal materials Tripterygium wilfordii Hook F (TwHF), has immunosuppressive, anti-inflammatory and anti-tumour effects. The purpose of this study was to examine whether triptolide has the neuroprotective effect on cerebral ischemia-reperfusion (I/R) injury and to explore its possible mechanism. Material and methods: The rat model of focal cerebral I/R was established by the suture-occluded method. The SD rats were randomly divided into five groups: sham operation group (Sham group), ischemia-reperfusion model group (I/R group), low concentration of triptolide group $(12.5 \mathrm{mg} / \mathrm{kg}, T L-L$ group), medium concentration of triptolide group (25 mg/kg, TL-M group) and high concentration of triptolide group (50 mg/kg, TL-H group). The neurological function of the rats was scored, the degree of brain oedema was detected by the dry-wet method, and the cerebral infarction area was determined by TTC staining. Nissl staining was used to detect neuronal damage. The contents of reactive oxygen species (ROS), malondialdehyde (MDA) and superoxide dismutase (SOD) were also detected. Meanwhile, the expression level of proteins related to Wnt/B-catenin signalling pathway was measured by Western blot.

Results: Compared with the I/R group, cerebral oedema, infarct volume, neurological impairment, the contents of MDA and ROS were reduced, while the SOD level was increased in the TL-L, TL-M, and TL-H groups. The results of Nissl staining showed that triptolide could reduce the nerve cell injury caused by cerebral I/R. In addition, the results of Western blot confirmed that the expression of Wnt1, $\beta$-catenin, c-Myc, and Cyclin-D1 were down-regulated after triptolide intervention, that is, inhibited the activation of Wnt/ $\beta$-catenin signalling pathway.

Conclusions: Triptolide mediates Wnt/ $\beta$-catenin signalling pathway to alleviate cerebral I/R injury in rats. This study provides ideas and experimental basis for the treatment of ischemic stroke patients.
\end{abstract}

Key words: cerebral ischemia/reperfusion, triptolide, neuroprotective, Wnt/ $\beta$-catenin signalling pathway.

\section{Introduction}

Cerebrovascular disease is one of the three fatal diseases in the world, with a high incidence, disability rate, mortality and recurrence rate [1]. Cerebrovascular disease mostly occurs in middle-aged and elderly people over 65 years old, and is the main disease leading to their death and disability. At present, the age of cerebrovascular disease's onset is getting younger, seriously endangering human health and quality of life [7]. According to statistics, ischemic vascular disease accounts for about $70-80 \%$ of cerebrovascu- 
lar diseases [25]. In China, there are 2 million new cerebral stroke patients every year, and about 1.5 million patients die of cerebral stroke every year, which is the third leading cause of death after heart disease and cancer [8]. Cerebrovascular disease brings the increasing mental and economic burden on the family and society. At present, cerebral ischemic disease is mainly treated through tissue recovery and recanalization and reperfusion to restore the blood supply of brain tissue [24]. However, there is clinical evidence that except for acute ischemic stroke, tissue recovery and recanalization and reperfusion often aggravate the damage of ischemic tissue, which not only cannot improve brain tissue ischemia, but also cause more serious damage to brain tissue and its function, causing cerebral ischemia-reperfusion (I/R) injury [16]. In recent years, the in-depth studies of the mechanisms of I/R injury have revealed that oxidative stress, metabolic disorder of energy, $\mathrm{Ca}^{2+}$ overload, and apoptosis play an important regulatory role in the process of cerebral I/R injury [16,20]. Accordingly, the pathogenesis of cerebral ischemic diseases, and effective preventive and therapeutic drugs have become the hot spot of medical research.

Tripterygium wilfordii Hook F (TwHF), also known as Lei Gong Teng (Thunder God Vine) is a vine shrub belonging to celastraceae [6]. As a traditional Chinese medicinal material, TwHF has anti-tumour, anti-inflammatory and immunosuppressive effects, is widely used in the treatment of a variety of inflammatory and autoimmune diseases (such as rheumatoid arthritis) [4]. Triptolide, the extract and the main active component of TwHF, has small molecular weight and lipophilicity, so it easily passes through the blood-brain barrier (BBB) and has a protective effect on the nervous system [35]. In both in vitro and in vivo experiments, triptolide has been shown to be effective in protecting damage of dopaminergic neurons induced by lipopolysaccharide (LPS) [27]. Meanwhile, triptolide can promote the synthesis of nerve growth factor (NGF), inhibit the aggregation and inflammation response of astrocytes, and promote the repair of spinal cord injury [31]. In addition, triptolide can also reduce the expression of COX-2 by inhibiting the NF- $\mathrm{KB}$ signalling pathway in astrocytes and inhibiting the activation of microglia [10]. However, the neuroprotective effect of triptolide on cerebral I/R injury is still unclear.

Previous studies have shown that $W n t / \beta$-catenin signalling pathway plays an important role in cerebral
I/R injury, involved in the proliferation, differentiation and axon formation and other processes of neural stem cells. Meanwhile, this signalling pathway is also critical in the formation and maintenance of bloodbrain barrier (BBB), cerebrovascular regeneration and remodelling [26]. Wnt protein is widely found in cerebral vessels and BBB, which can participate in the regulation of cerebral angiogenesis and BBB differentiation. Wnt/ $\beta$-catenin signalling pathway is of great significance in injury repair and neurovascular remodelling in ischemic stroke [26]. Many researches have confirmed that cerebral I/R injury can activate $W n t / \beta$-catenin signalling pathway, but whether the activation of this pathway protects or injures the tissues is controversial [23]. In the rat model of cerebral $I / R$ injury induced by the middle cerebral artery, the expression of $\mathrm{Wnt} / \beta$-catenin signalling pathway was up-regulated, and the upstream and downstream genes APC, c-Myc and Cyclin-D1 of $\beta$-catenin were up-regulated in this process, which could promote further apoptosis of nerve cells [21]. Therefore, we speculate that $W n t / \beta$-catenin signalling pathway plays an important role in cerebral I/R injury.

Traditional Chinese medicine (TCM) has the characteristics of multi-component, multi-target and multi-pathway medicine. Studies have confirmed that many kinds of TCM can protect nervous system diseases. Some TCM play a neural role through $W n t / \beta$-catenin signalling pathway, and its mechanism is to regulate the function of the nervous system after cerebral ischemia by affecting the expression of proteins, such as Wnt1, c-Myc, Cyclin-D1, $\beta$-catenin. And the above evidence can also indicate that the $W n t / \beta$-catenin signalling pathway performs biological function in the disease. Therefore, we speculate that triptolide may alleviate cerebral I/R injury in rats through Wnt/ $\beta$-catenin signalling pathway. Thus, we will carry out research on this speculation so as to provide a new idea for the treatment of cerebral I/R injury.

\section{Material and methods \\ Experimental animals and grouping}

Twenty-five 8-week-old healthy male SD rats, weighing $225 \mathrm{~g}$ to $245 \mathrm{~g}$ were selected as study subjects. The ambient temperature of the experiment was $22 \pm 1^{\circ} \mathrm{C}$ and the light time was 12 hours/ day. The above rats were randomly divided into 5 groups $(n=5)$ : sham operation group (Sham group), 
ischemia-reperfusion model group (I/R group), low concentration of triptolide (Merck, Germany) group (12.5 mg/kg, TL-L group), medium concentration of triptolide group ( $25 \mathrm{mg} / \mathrm{kg}$, TL-M group) and high concentration of triptolide group $(50 \mathrm{mg} / \mathrm{kg}, \mathrm{TL}-\mathrm{H}$ group). In the I/R and triptolide treated groups the suture-occluded method was used to block the middle cerebral artery. In the Sham group, only internal and external cervical vessels were separated and no suture to block. In the TL-L, TL-M, TL-H groups, the rats were injected intraperitoneally with an appropriate amount of DMSO solvent mixed with triptolide at 24 hours before operation. The Sham group and the I/R group were also injected intraperitoneally with the same dose of DMSO solvent as in the triptolide treated groups before the operation.

\section{Establishment of rat models}

The SD rat models of focal cerebral I/R injury were established [18]. The rats were weighed and anaesthetized with intraperitoneal injection of $4 \%$ chloral hydrate at a rate of $8 \mathrm{ml} / \mathrm{kg}$. The rat was placed supine and fixed, and then a longitudinal incision was made in the middle of the neck. The right common carotid artery, internal carotid artery and external carotid artery were separated and fully exposed. Suture was used to ligate the right proximal common carotid artery and the base of the external carotid artery, respectively. An incision was made at the right common carotid artery and then the head end of the suture was burnt into a round shape $(0.25 \mathrm{~mm}$ in diameter), which then was inserted into the right common carotid artery $(17 \mathrm{~mm}$ in diameter). After 2 hours of ischemia, the suture was pulled out slowly. Next, the distal right common carotid artery was ligated to form reperfusion injury.

\section{Neurobehavioral symptom score}

At 24 hours after $I / R$, the rats were scored for neurological deficits in limb function $[12,19]$. The scoring criteria were as follows: 1) 0 points: rats without obvious symptoms of neurological impairment; 2) 1 point: the left front paw is difficult to fully extend; 3) 2 points: lean to the left side when walking; 4) 3 points: unable to walk; and 5) 4 points: die.

\section{Determination of brain water content}

The dry-wet method was used. The rats were killed at the specified time phases, and their brains were removed by decapitation. The brain tissues of both cerebral hemispheres $(L-$ left hemisphere, $\mathrm{R}$ - right hemisphere) were taken, respectively. First, the wet weight was measured using an electronic balance. Then the samples were dried in an infrared drying box at $95^{\circ} \mathrm{C}$ for 24 to 48 hours to obtain constant weight, and the dry weight was determined. Brain water content $=($ wet weight - dry weight $) /$ wet weight $\times 100 \%$.

\section{Triphenyl tetrazolium chloride staining to detect the cerebral infarction area}

24 hours after $I / R$, the rats in each group were injected with chloral hydrate $(3 \mathrm{ml} / \mathrm{kg}$ ) for anaesthesia, and their brains were taken. Subsequently, the surface blood of the brains was rinsed with normal saline, then dried with filter paper, and quickly frozen at $-20^{\circ} \mathrm{C}$ for $20 \mathrm{~min}$. Next, the brain sections $(2 \mathrm{~mm})$ were sliced using brain matrices. The sections were stained with $2 \%$ triphenyl tetrazolium chloride (TTC), placed in an oven at $37^{\circ} \mathrm{C}$ and stained for $30 \mathrm{~min}$ in the dark. Normal brain tissues were red, while the areas of cerebral infarction were unstained or white. Finally, the sections were placed on slides and photographed. The infarct area was measured and calculated using Image J.

\section{Nissl staining}

Nissl staining is a method for staining nerve tissue using alkaline dyes, which is mainly combined with nucleic acid in nerve tissues. At 24 hours after $I / R$, the rats in each group were perfused to extract the tissues. During the extraction, the tissues were prevented from being squeezed to avoid the false appearance of neuronal contraction. Frozen tissue sections were washed twice with distilled water for 30 seconds each time. Then the sections were stained with $0.5 \%$ toluidine blue at $50-60^{\circ} \mathrm{C}$ for 30 min, washed twice using distilled water, and differentiated with $95 \%$ ethanol for 5 seconds. Next, the sections were dehydrated by gradient alcohol and made transparent using xylene. Finally, the slices were sealed by neutral balsam.

\section{ELISA to detect TNF- $\alpha$, IL-6, IL-8 and IL-1 $\beta$ in brain tissue}

The specific operation steps were carried out according to the instruction of the ELISA detection kit for interleukin (IL)-1 $\beta$, tumour necrosis factor $\alpha$ 
(TNF- $\alpha$ ), IL-6 and IL-8. After subtracting the blank value from all OD values, the standard curve was established with the OD values of 1000, 500, 250, $125,62,31,16,0 \mathrm{ng} / \mathrm{l}$ standard samples using curve expert 1.3 software. The contents of TNF, IL-6, IL-8 and IL-1 were calculated according the OD values of the experimental samples.

\section{Determination of SOD, ROS and MDA in brain tissue}

The brain tissues of rats in each group were collected and after homogenate, the activity of superoxide dismutase (SOD), the contents of reactive oxygen species (ROS) and malondialdehyde (MDA) were determined. Superoxide dismutase assay kit, active oxygen assay kit, and MDA determination kit (Nanjing Jiancheng Bioengineering Institute, Jiangsu, China) were used to detect the activity of SOD and the contents of ROS and MDA according to the instructions.

\section{Western blot to detect Wnt/ $\beta$-catenin signalling pathway-related proteins}

At 24 hours after I/R, the SD rats in each group were anesthetized to decapitate. The brain tissues on the ischemic side were quickly collected and stored at $-80^{\circ} \mathrm{C}$ for use. The total protein of the samples in each group was extracted by RIPA lysis buffer, and the total protein concentration was determined using BCA method and then was adjusted to the same in each group. The separating gel and stacking gel were prepare. The protein loaded in the gel was first separated using SDS-PAGE and then transferred to PVDF membrane. Following the blocking step with TBST blocking buffer containing 5\% skim milk for 2 hours, the membrane was incubated overnight at $4^{\circ}$ with the following primary antibodies: rabbit polyclonal antibody Wnt1 (ab15251, 1 : 1000), rabbit monoclonal antibody $\beta$-catenin (ab32572, $1: 1000)$, rabbitmonoclonalantibodyc-Myc (ab32072, 1 : 1000), rabbit monoclonal antibody Cyclin-D1 (ab134175, $1: 1000$ ) and rabbit monoclonal antibody GAPDH (ab181602, 1 : 1000). Subsequently, the membrane was incubated with the secondary antibodies labelled with horse radish peroxidase (HRP) for 2 hours. The target proteins were developed by chemiluminescence, and the results were analysed by the gel imaging system. The image was then quantized using Image J (National Institutes of Health).

\section{Statistical analysis}

SPSS 22.0 software was used for statistical analysis. The measurement data were expressed as mean \pm standard deviation (SD). The comparisons between the two groups were performed using $T$-test, while the comparisons of multiple groups using one-way analysis of variance (ANOVA). Each experiment was repeated 3 times. $P<0.05$ was considered significant.

\section{Results}

\section{Triptolide reduces neurological deficit in rats with cerebral I/R}

To investigate the protective effect of triptolide on the neurological function of $I / R$, the neurological deficit in rats at 24 hours after reperfusion was scored. The results (Fig. 1) showed that compared with the Sham group, the neurological deficit score was significantly increased in the I/R group. Compared with the I/R group, the scores of TL-L, TL-M and $\mathrm{TL}-\mathrm{H}$ groups were decreased significantly, and in a concentration-dependent manner. The results suggested that triptolide could promote neurological repair in the rats with cerebral I/R injury.

\section{Triptolide reduces cerebral oedema and infarct area in rats with cerebral I/R}

The results of the brain oedema test (Fig. 2A) showed that the water content of brain tissue in the

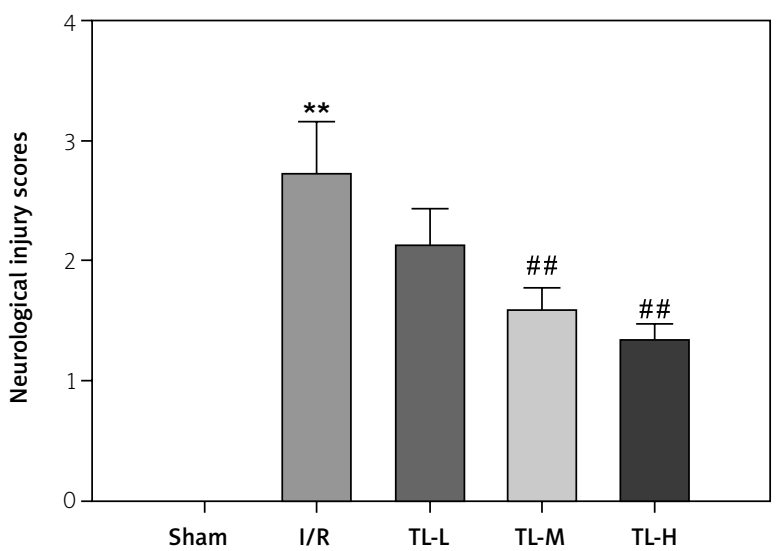

Fig. 1. Effect of triptolide on the neurological deficit in rats with cerebral I/R. At 24 hours after reperfusion, the neurological deficit of rats in each group was scored. ${ }^{*} p<0.05$ vs. Sham group; ${ }^{\# \#} p<0.01$ vs. I/R group. I/R - ischemia-reperfusion, TL-L-12.5 mg/kg triptolide, TL-M- $25 \mathrm{mg} / \mathrm{kg}$ triptolide, $\mathrm{TL}-\mathrm{H}-50 \mathrm{mg} / \mathrm{kg}$ triptolide. 

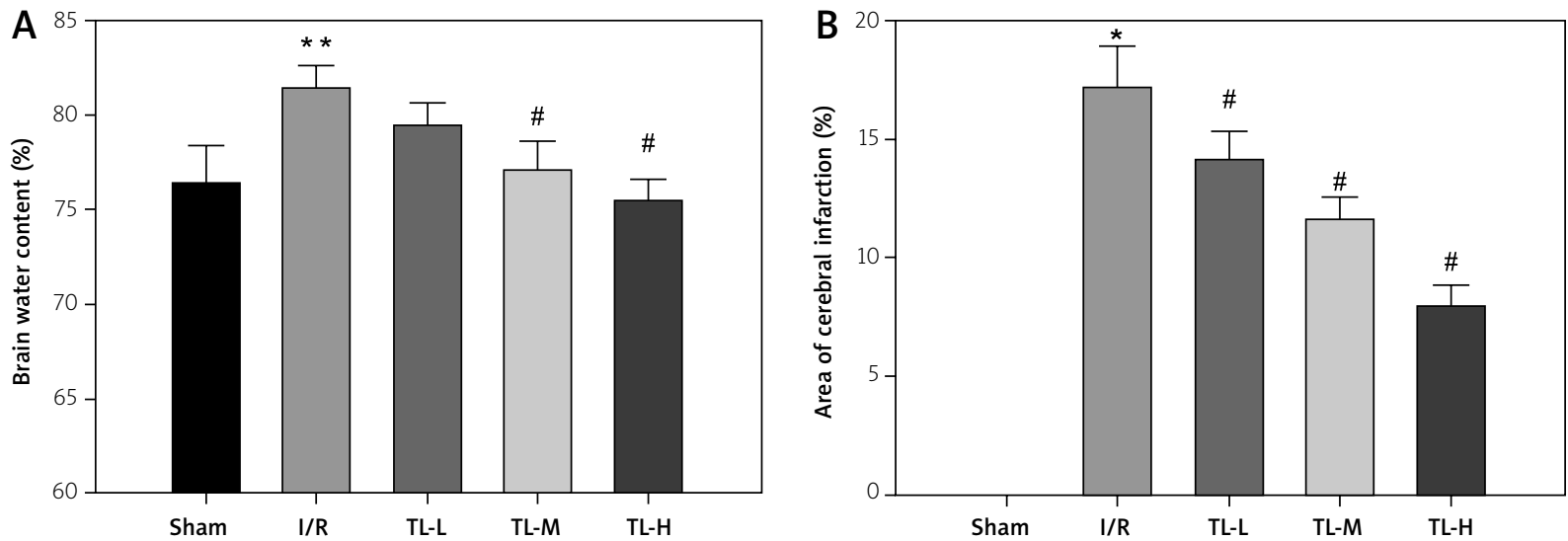

Fig. 2. Effects of triptolide on brain function in rats with I/R. At 24 hours after reperfusion, the water content of brain tissue (A) and infarct area (B) in rats were measured. ${ }^{*} p<0.05$ vs. Sham group; ${ }^{*} p<0.05$ vs. I/R group. I/R - ischemia-reperfusion, TL-L $-12.5 \mathrm{mg} / \mathrm{kg}$ triptolide, TL-M - $25 \mathrm{mg} / \mathrm{kg}$ triptolide, $\mathrm{TL}-\mathrm{H}-50 \mathrm{mg} / \mathrm{kg}$ triptolide.

I/R group was significantly increased compared with the Sham group. Compared with the I/R group, the water content of the brain tissue in the TL-L, TL-M, $\mathrm{TL}-\mathrm{H}$ groups was decreased and in a concentration-dependent manner $(p<0.05)$.

At 24 hours after I/R, the results of TTC staining (Fig. 2B) showed that there was no infarct in the brain tissue of rats in the Sham group. Compared with the Sham group, the cerebral infarction area in the I/R group was significantly larger $(p<0.05)$. Compared with the I/R group, the cerebral infarction area of the TL-L, TL-M, and TL-H groups was significantly reduced ( $p<0.05$ ), and in a concentration-dependent manner. The results suggested that triptolide reduced cerebral oedema and infarct area in the rats with cerebral $I / R$, so as to reduce or delay the pathological process of reperfusion injury.

\section{Changes in Nissl staining of neurons in rats with cerebral I/R after triptolide intervention}

The results of the Nissl staining (Fig. 3) showed that the vertebral cells in the hippocampal region of all rats in the Sham group arranged neatly and compactly, with abundant and deeply stained Nissl bodies. Compared with the Sham group, the neurons in the hippocampal region in the I/R group were sparse, with decreased Nissl bodies and a massive proliferation of glial cells. Compared with the I/R group, glial cells were proliferated, the number of neurons and Nissl bodies was reduced in the TL-L, TL-M, and TL-H groups, but the degree was alleviated compared with the I/R group; the higher the TL concentration, the lower the degree of neuronal injury.

\section{Triptolide inhibits inflammatory response in rats after I/R}

The results of ELISA (Fig. 4) showed that, compared with the Sham group, the expression levels of TNF- $\alpha$, IL- 6 , IL- 8 and IL- $1 \beta$ in the brain tissue of rats in the I/R group were significantly elevated. Compared with the I/R group, the expression levels of TNF- $\alpha$, IL-6, IL-8 and IL-1 $\beta$ in TL-L, TL- $M, T L-H$ groups were decreased in a concentration-dependent manner.

\section{Triptolide improves the ability of} antioxidation in rats with cerebral I/R

Compared with the rats in the Sham group, the levels of ROS and MDA were significantly increased while the level of SOD was significantly decreased in the I/R group $(p<0.05)$. Compared with the I/R group, the levels of ROS and MDA in TL-L, TL-M and $\mathrm{TL}-\mathrm{H}$ groups decreased significantly, while the level of SOD increased obviously $(p<0.05)$, and in a concentration-dependent manner (Fig. 5).

\section{Triptolide inhibits Wnt/ $\beta$-catenin signalling pathway in brain tissue of rats with cerebral I/R}

In order to verify the molecular mechanism of triptolide on the repair of brain injury in rats with cerebral I/R, we studied the effect of triptolide on 
Sham

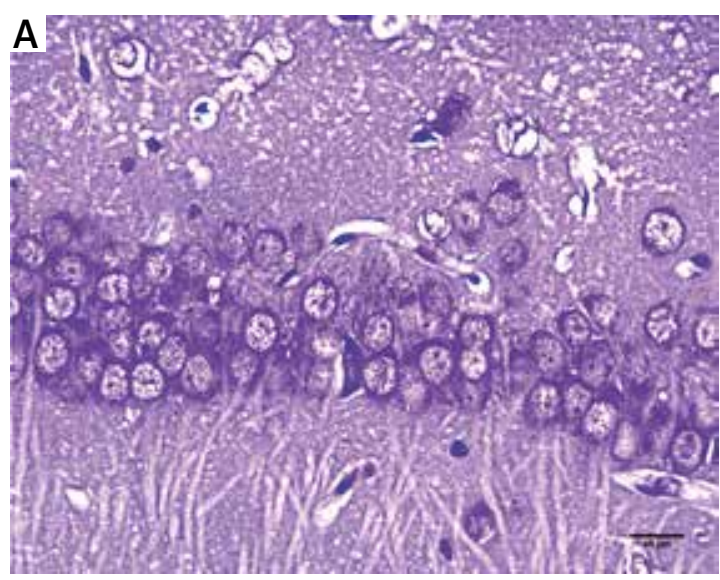

TL-L
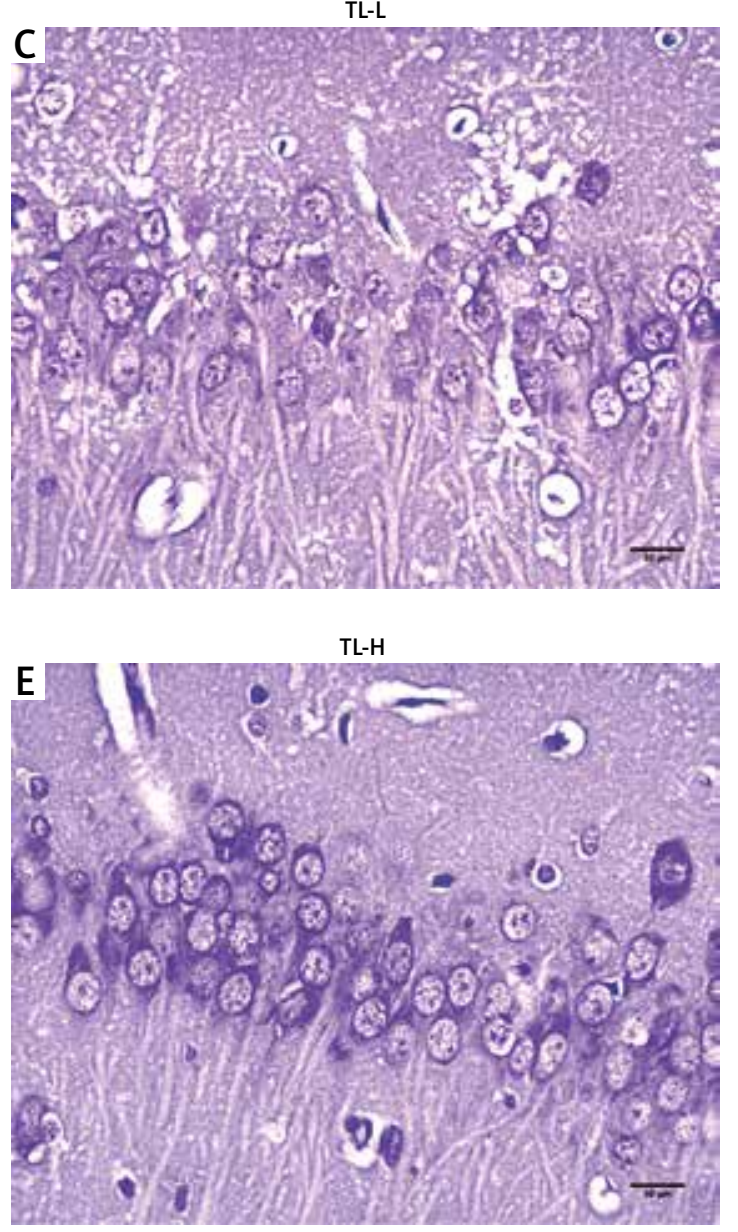

Wnt/ $\beta$-catenin signalling pathway. The results of Western blot (Fig. 6) showed that, compared with the Sham group, the expression levels of Wnt1, $\beta$-catenin, c-Myc and Cyclin-D1 in the brain tissue of rats in the I/R group were significantly increased. Compared with the I/R group, the expression lev-
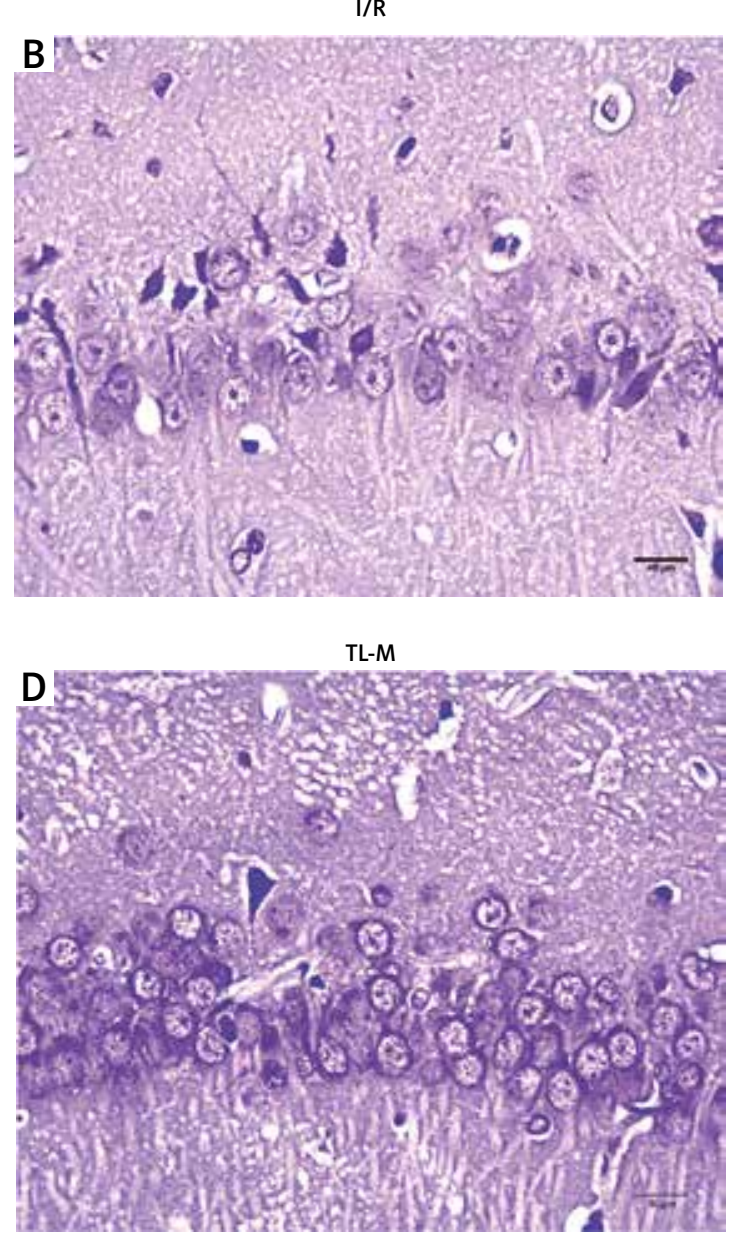

Fig. 3. Protective effects of triptolide on neurons in rats with cerebral I/R. Nissl staining (magnification, 200x) to detect nerve tissue damage in rats. I/R - ischemia-reperfusion, TL-L $-12.5 \mathrm{mg} / \mathrm{kg}$ triptolide, TL-M - $25 \mathrm{mg} / \mathrm{kg}$ triptolide, TL-H - $50 \mathrm{mg} / \mathrm{kg}$ triptolide.

els of Wnt1, $\beta$-catenin, C-Myc and Cyclin-D1 in TL-L, TL-M and TL-H groups were significantly reduced in a concentration-dependent manner $(p<0.05)$. These results suggested that triptolide could repair brain injury in rats with cerebral $1 / R$ by regulating $W n t / \beta$-catenin signalling pathway. 


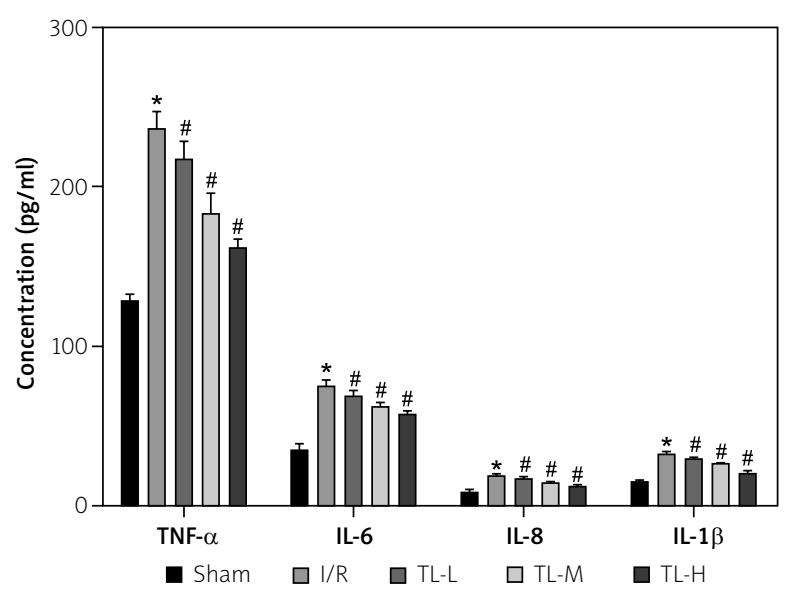

Fig. 4. Effect of triptolide on inflammation in rats with cerebral I/R. At 24 hours after reperfusion, the brain tissues of rats were collected and the expressions of inflammatory factors TNF- $\alpha$, IL-6, IL- 8 and IL-1 $\beta$ were detected by ELISA. ${ }^{*} p<0.05$ vs. Sham group; ${ }^{\#} p<0.05$ vs. I/R group. I/R - ischemia-reperfusion, TL-L - $12.5 \mathrm{mg} / \mathrm{kg}$ triptolide, TL-M - $25 \mathrm{mg} / \mathrm{kg}$ triptolide, TL-H $50 \mathrm{mg} / \mathrm{kg}$ triptolide.
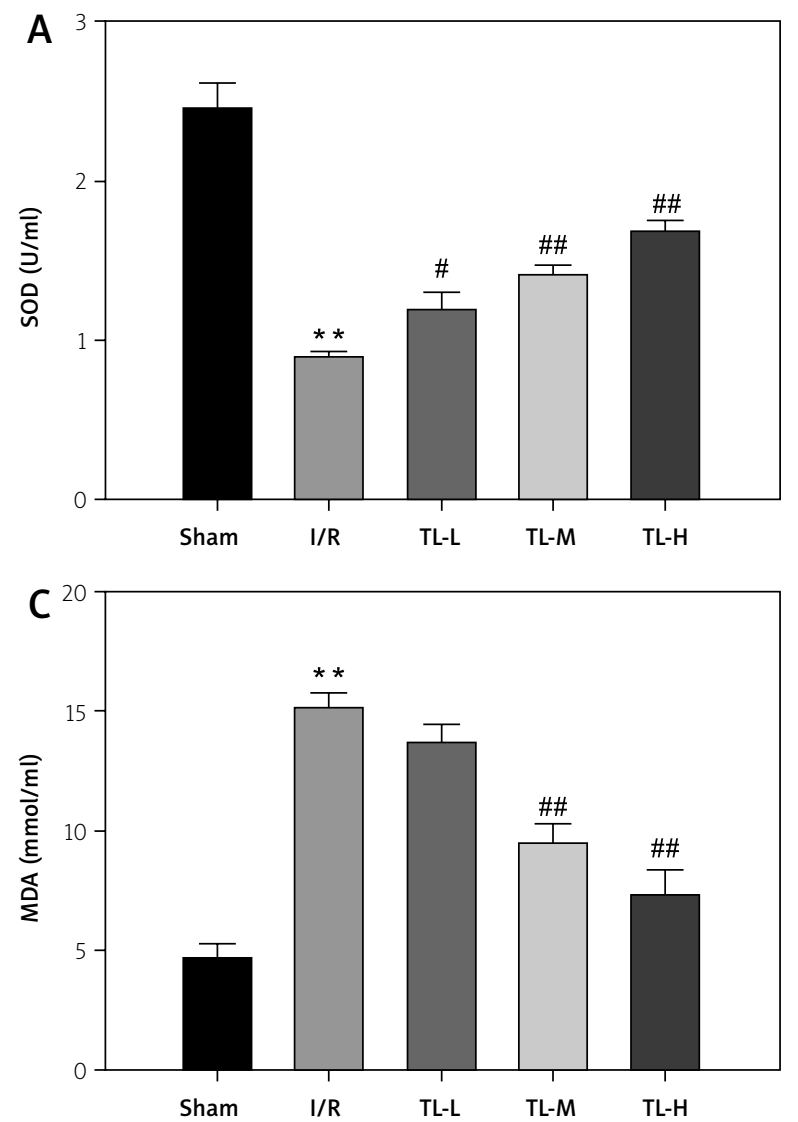

\section{Discussion}

Tripterygium wilfordii Hook $\mathrm{F}$ has a variety of pharmacological activities such as improving microcirculation, immunomodulation, dilating blood vessels, inhibition of platelet aggregation, and antitumor activity [28]. A large amount of literature has confirmed that triptolide is the main active compound of TwHF, with the effects of anti-inflammation and immunodepression. Triptolide is now clinically used for the treatment of inflammatory and autoimmune diseases such as rheumatoid arthritis, systemic lupus erythematosus, and kidney disease [29]. Current pharmacological studies have shown that triptolide also has a certain therapeutic effect on ischemic stroke [2]. Hao et al. [15] found that triptolide could reduce the cytotoxicity and apoptosis induced by OGD, and inhibit the activation of NF- $\mathrm{KB}$ and $\mathrm{p} 38$ MAPK induced by TNF- $\alpha$ in vitro, while triptolide also had a neuroprotective effect on rat models with middle cerebral artery embolization in vivo. It has also been reported that the neurological deficit is improved and the number of neu-

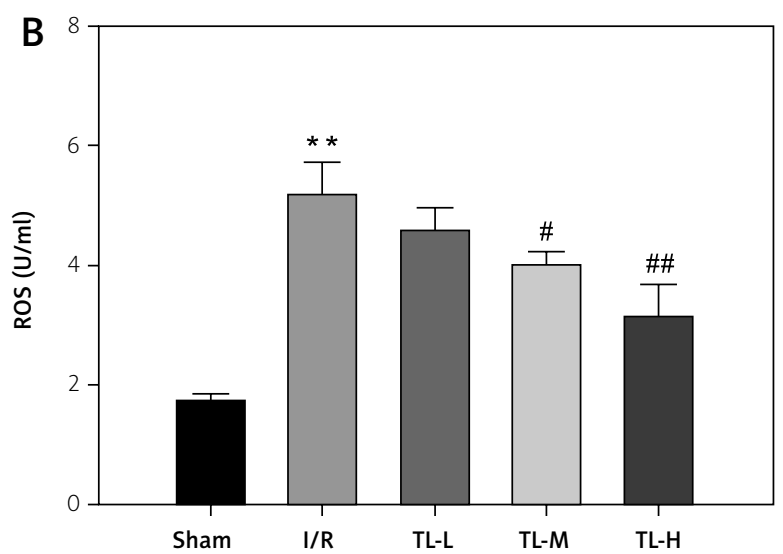

Fig. 5. Effects of triptolide on the activities of SOD, ROS and MDA in rats with cerebral ischemia-reperfusion. ${ }^{*} p<0.05$ vs. Sham group; ${ }^{\#} p<0.05$ and ${ }^{\# \#} p<0.01$ vs. I/R group. I/R - ischemia-reperfusion, TL-L - $12.5 \mathrm{mg} / \mathrm{kg}$ triptolide, TL-M - $25 \mathrm{mg} / \mathrm{kg}$ triptolide, TL-H - $50 \mathrm{mg} / \mathrm{kg}$ triptolide. 

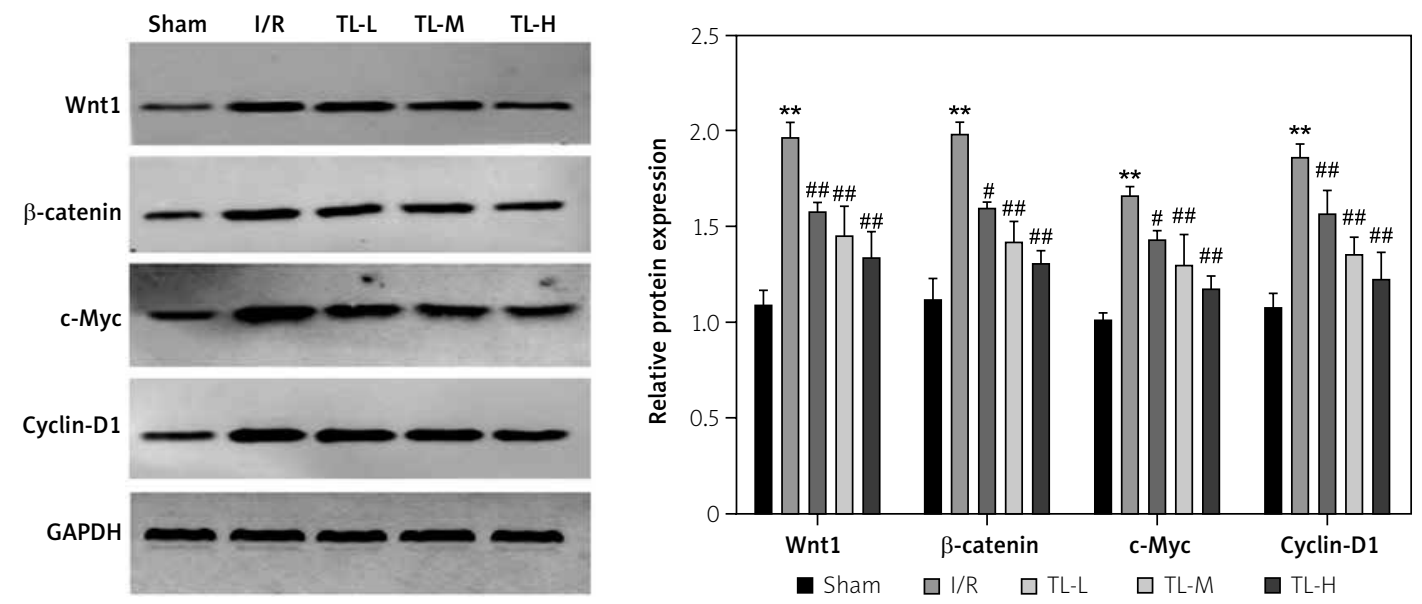

Fig. 6. Effect of triptolide on Wnt/ $\beta$-catenin signalling pathway in brain tissue of rats with cerebral I/R. Western blot was used to detect the protein expression levels of Wnt1, $\beta$-catenin, c-Myc and Cyclin-D1 in the rat model of cerebral I/R injury after treated with different doses of triptolide. ${ }^{*} p<0.05$ vs. Sham group; ${ }^{\#} p<0.05$ and \#\#p $<0.01$ vs. I/R group. I/R - ischemia-reperfusion, TL-L $-12.5 \mathrm{mg} / \mathrm{kg}$ triptolide, TL-M $25 \mathrm{mg} / \mathrm{kg}$ triptolide, $\mathrm{TL}-\mathrm{H}-50 \mathrm{mg} / \mathrm{kg}$ triptolide.

trophils infiltration and neuronal apoptosis were reduced in the rat MCAO models by down-regulating the expression of iNOS, COX-2, GFAP and NF-KB, elevating $\mathrm{BCl}-2$ expression and reducing the expression of Caspase-3 [2]. In addition, Li et al. also found that triptolide exerts neuroprotective effects in cerebral ischemia by regulating cellular autophagy and apoptosis of signalling pathways [17]. In this study, the rat model of focal cerebral I/R was established by the suture-occluded method, so as to investigate the therapeutic mechanism of triptolide. The results of this study confirmed that, compared with the I/R group, the neurological impairment score, cerebral infarction area, neuron injury and brain oedema in rats with cerebral I/R in the TL-L, TL-M, TL-H groups were decreased significantly and in a concentration-dependent manner, indicating that triptolide can significantly slow down the brain injury induced by $I / R$, which is consistent with the above literature.

A large number of studies have confirmed that a large number of ROS would generate, which could increase the content of MDA and consume SOD to decrease its activity during the acute phase of cerebral I/R in rats [3]. And the high production of free radicals is the main cause of delayed neuronal damage. For one thing, cerebral I/R leads to new oxygen supply to ischemic tissue, which activates xanthine oxidase, produces superoxide anion free radicals and attacks neuronal membranes and microvessels rich in double bonds of unsaturated fatty acids, causing chain reaction of lipid peroxidation and disturbance of microcirculation, thus resulting in a large number of neuronal damage. For another thing, cerebral I/R continues the conversion from alkane radicals to MDA, which are stopped due to oxygen depletion during ischemia, resulting in a new vicious circle. At this time, the antioxidants in the body are largely consumed by free radicals, and the free radicals produced by the reaction have exceeded the body's ability to remove them; the rapid accumulation of free radicals in ischemic tissues certainly further attack the biofilm structure of other cells, causing more cell necrosis [13,22,32]. This study showed that triptolide significantly increased the content of SOD to reduce the production of ROS and to reduce the level of MDA after cerebral I/R in rats. It is suggested that triptolide has the effect of scavenging free radicals, improving the ability of antioxidation, reducing the production of free radicals and blocking its chain reaction during cerebral $I / R$, so as to protect ischemic brain tissue from free radical damage.

There are various mechanisms of $1 /$ R-induced injury. Pro-inflammatory response, like ROS, also plays an important role in the pathogenesis of $I / R$ [5]. Reducing the pro-inflammatory response can significantly reduce I/R-induced injury [11]. In the present study, we found that the expression of pro-inflammatory factors TNF- $\alpha$, IL-6, IL-8, and IL-1 $\beta$ in the brain tissue of I/R rats are all up-regulated, and triptolide could inhibit their expression. Studies 
have shown that the Wnt/ $\beta$-catenin pathway also plays an important role in the expression of various inflammatory mediators [9]. The activation of Wnt3a in mouse microglia leads to the expression and release of IL-6, IL-12 and interferon [14]. Meanwhile, $W n t / \beta$-catenin signalling pathway is involved in the development of BBB, and its regulatory disorder will lead to the destruction of BBB $[30,34]$. In addition, the activation of $W n t / \beta$-catenin signalling pathway could promote nerve regeneration and nerve function recovery after focal cerebral ischemia. Wnt1, $\beta$-catenin, c-Myc and Cyclin-D1 are the key factors of Wnt/ $\beta$-catenin signalling pathway [33]. The significant activation of the above proteins was found after cerebral ischemia, while the activation degree of Wnt $/ \beta$-catenin signalling pathway was able to be reduced after intervention with different doses of triptolide.

In conclusion, triptolide significantly reduces the neurological impairment score, cerebral infarct size and cerebral oedema, increases SOD activity, decreases the content of MDA and ROS, reduces neuronal injury, and down-regulates the protein expression of Wnt1, $\beta$-catenin, c-Myc and Cyclin-D1 in $W n t / \beta$-catenin signalling pathway. These results indicate that the neuroprotective effect of triptolide on cerebral I/R rats may be achieved by regulating the expression of proteins related to Wnt/ $\beta$-catenin signalling pathway. The conclusions of this study need to be verified by follow-up animal experiments and clinical tissue specimens, which are worthy of a systematic in-depth study to provide experimental evidence for the treatment of cerebral I/R injury diseases.

\section{Acknowledgements}

The authors thank Guangzhou Yujia Biotechnology Co., Ltd. for excellent technical assistance.

\section{Disclosure}

The authors report no conflict of interest.

\section{References}

1. Agostoni EC, Longoni M. Migraine and cerebrovascular disease: still a dangerous connection? Neurol Sci 2018; 39: 33-37.

2. Bai S, Hu Z, Yang Y, Yin Y, Li W, Wu L, Fang M. Anti-inflammatory and neuroprotective effects of triptolide via the NF-kappaB signaling pathway in a rat MCAO model. Anat Rec (Hoboken) 2016; 299: 256-266.
3. Bo Z, Huiling L. Mangiferin improves memory deficits followed cerebral ischemia reperfusion in mice through attenuation of oxidative stress and AChE activity. Lat Am J Pharm 2015; 34: 1229-1238.

4. Chen H, Chen Q, Jiang CM, Shi GY, Sui BW, Zhang W, Yang LZ, Li ZY, Liu L, Su YM, Zhao WC, Sun HQ, Li ZZ, Fu Z. Triptolide suppresses paraquat induced idiopathic pulmonary fibrosis by inhibiting TGFB1-dependent epithelial mesenchymal transition. Toxicol Lett 2018; 284: 1-9.

5. Chen LW, Egan L, Li ZW, Greten FR, Kagnoff MF, Karin M. The two faces of IKK and NF-kappaB inhibition: prevention of systemic inflammation but increased local injury following intestinal ischemia-reperfusion. Nat Med 2003; 9: 575-581.

6. Chen SR, Dai Y, Zhao J, Lin L, Wang Y, Wang Y. A mechanistic overview of triptolide and celastrol, natural products from Tripterygium wilfordii Hook F. Front Pharmacol 2018; 9: 104.

7. Crawford KM, Gallego-Fabrega C, Kourkoulis C, Miyares L, Marini S, Flannick J, Burtt NP, von Grotthuss M, Alexander B, Costanzo MC, Vaishnav NH, Malik R, Hall J, Chong M, Rosand J, Falcone GJ. Cerebrovascular disease knowledge portal: an open-access data resource to accelerate genomic discoveries in stroke. Stroke 2018; 49: 470-475.

8. Dong R, Dong Z, Liu H, Shi F, Du J. Prevalence, risk factors, outcomes, and treatment of obstructive sleep apnea in patients with cerebrovascular disease: a systematic review. J Stroke Cerebrovasc Dis 2018; 27: 1471-1480.

9. Fang Y, Kang Y, Zou H, Cheng X, Xie T, Shi L, Zhang H. Beta-elemene attenuates macrophage activation and proinflammatory factor production via crosstalk with Wnt/beta-catenin signaling pathway. Fitoterapia 2018; 124: 92-102.

10. Geng Y, Fang M, Wang J, Yu H, Hu Z, Yew DT, Chen W. Triptolide down-regulates COX-2 expression and PGE2 release by suppressing the activity of NF-kappaB and MAP kinases in lipopolysaccharide-treated PC12 cells. Phytother Res 2012; 26: 337-343.

11. Ghyczy M, Torday C, Kaszaki J, Szabo A, Czobel M, Boros M. Oral phosphatidylcholine pretreatment decreases ischemia-reperfusion-induced methane generation and the inflammatory response in the small intestine. Shock 2008; 30: 596-602.

12. Gonzalez-Falcon A, Candelario-Jalil E, Garcia-Cabrera M, Leon OS. Effects of pyruvate administration on infarct volume and neurological deficits following permanent focal cerebral ischemia in rats. Brain Res 2003; 990: 1-7.

13. Guo F, Song W, Jiang T, Liu L, Wang F, Zhong H, Yin H, Wang Q, Xiong L. Electroacupuncture pretreatment inhibits NADPH oxidase-mediated oxidative stress in diabetic mice with cerebral ischemia. Brain Res 2014; 1573: 84-91.

14. Gustafson B, Smith U. Cytokines promote Wnt signaling and inflammation and impair the normal differentiation and lipid accumulation in 3T3-L1 preadipocytes. J Biol Chem 2006; 281: 9507-9516.

15. Hao M, Li X, Feng J, Pan N. Triptolide protects against ischemic stroke in rats. Inflammation 2015; 38: 1617-1623.

16. Hou Y, Wang Y, He Q, Li L, Xie H, Zhao Y, Zhao J. Nrf2 inhibits NLRP3 inflammasome activation through regulating Trx1/ TXNIP complex in cerebral ischemia reperfusion injury. Behav Brain Res 2018; 336: 32-39. 
17. Li W, Yang Y, Hu Z, Ling S, Fang M. Neuroprotective effects of DAHP and Triptolide in focal cerebral ischemia via apoptosis inhibition and PI3K/Akt/mTOR pathway activation. Front Neuroanat 2015; 9: 48.

18. Lin R, Huan-Sen H, Wen-Xiang J, Hai-Ming C, Xiong-Juan L, QingJuan G. Tetrandrine attenuated cerebral ischemia/reperfusion injury and induced differential proteomic changes in a MCAO mice model using 2-D DIGE. Neurochem Res 2013; 38: 1871-1879.

19. Longa EZ, Weinstein PR, Carlson S, Cummins R. Reversible middle cerebral artery occlusion without craniectomy in rats. Stroke 1989; 20: 84-91.

20. Lou Z, Wang AP, Duan XM, Hu GH, Song GL, Zuo ML, Yang ZB. Upregulation of NOX2 and NOX4 mediated by TGF-beta signaling pathway exacerbates cerebral ischemia/reperfusion oxidative stress injury. Cell Physiol Biochem 2018; 46: 2103-2113.

21. Scott EL, Brann DW. Estrogen regulation of Dkk1 and Wht/ beta-Catenin signaling in neurodegenerative disease. Brain Res 2013; 1514: 63-74.

22. Song Y, Wang LB, Bei Y, Qin DX, Ai LY, Ma QZ, Lin PY. Carvacryl acetate, a semisynthetic monoterpenic ester obtained from essential oils, provides neuroprotection against cerebral ischemia reperfusion-induced oxidative stress injury via the Nrf2 signalling pathway. Food Funct 2020; 11: 1754-1763.

23. Sun FL, Wang W, Zuo W, Xue JL, Xu JD, Ai HX, Zhang L, Wang XM, Ji XM. Promoting neurogenesis via Wnt/beta-catenin signaling pathway accounts for the neurorestorative effects of morroniside against cerebral ischemia injury. Eur J Pharmacol 2014; 738: 214-221.

24. Tian T, Zhang HX, He CP, Fan S, Zhu YL, Qi C, Huang NP, Xiao ZD, Lu ZH, Tannous BA, Gao J. Surface functionalized exosomes as targeted drug delivery vehicles for cerebral ischemia therapy. Biomaterials 2018; 150: 137-149.

25. Wang J, Zhang L, Liu B, Wang Q, Chen Y, Wang Z, Zhou J, Xiao W, Zheng C, Wang Y. Systematic investigation of the Erigeron breviscapus mechanism for treating cerebrovascular disease. J Ethnopharmacol 2018; 224: 429-440.

26. Wang W, Li M, Wang Y, Li Q, Deng G, Wan J, Yang Q, Chen Q Wang J. GSK-3beta inhibitor TWS119 attenuates rtPA-induced hemorrhagic transformation and activates the Wnt/beta-catenin signaling pathway after acute ischemic stroke in rats. Mol Neurobiol 2016; 53: 7028-7036.

27. Wei D, Huang Z. Anti-inflammatory effects of triptolide in LPS-induced acute lung injury in mice. Inflammation 2014; 37: 1307-1316.

28. Wong KF, Yuan Y, Luk JM. Tripterygium wilfordii bioactive compounds as anticancer and anti-inflammatory agents. Clin Exp Pharmacol Physiol 2012; 39: 311-320.

29. Xi C, Peng S, Wu Z, Zhou Q, Zhou J. Toxicity of triptolide and the molecular mechanisms involved. Biomed Pharmacother 2017; 90: 531-541.

30. Xing Y, Zhang X, Zhao K, Cui L, Wang L, Dong L, Li Y, Liu Z, Wang C, Zhang X, Zhu C, Qiao H, Ji Y, Cao X. Beneficial effects of sulindac in focal cerebral ischemia: a positive role in Wnt/ beta-catenin pathway. Brain Res 2012; 1482: 71-80.

31. Xue B, Jiao J, Zhang L, Li KR, Gong YT, Xie JX, Wang XM. Triptolide upregulates NGF synthesis in rat astrocyte cultures. Neurochem Res 2007; 32: 1113-1119.
32. Ye Y, Jianzeng G, Junqing Y, Qixin Z. Electroacupuncture pretreatment inhibits Effects of heme oxygenase-1 on the brain damage induced by cerebral ischemia/reperfusion. Chinese Pharmacological Bulletin 2008; 1200-1204.

33. Yoshida T, Sopko NA, Kates M, Liu X, Joice G, McConkey DJ, Bivalacqua TJ. Three-dimensional organoid culture reveals involvement of Wnt/beta-catenin pathway in proliferation of bladder cancer cells. Oncotarget 2018; 9: 11060-11070.

34. Zhan L, Liu D, Wen H, Hu J, Pang T, Sun W, Xu E. Hypoxic postconditioning activates the Wnt/beta-catenin pathway and protects against transient global cerebral ischemia through Dkk1 Inhibition and GSK-3beta inactivation. FASEB J 2019; 33: 9291 9307.

35. Zhang L, Chang J, Zhao Y, Xu H, Wang T, Li Q, Xing L, Huang J, Wang $Y$, Liang Q. Fabrication of a triptolide-loaded and poly-gamma-glutamic acid-based amphiphilic nanoparticle for the treatment of rheumatoid arthritis. Int I Nanomedicine 2018; 13: 2051-2064. 